\title{
Systematic screening for PRKAR1A gene rearrangement in Carney complex: identification and functional characterization of a new in-frame deletion
}

\author{
M Guillaud Bataille, ${ }^{1,2, *}$ Y Rhayem ${ }^{1,2, *}$, S B Sousa ${ }^{6,7}$, R Libé $^{3}$, M Dambrun ${ }^{1}$, \\ C Chevalier ${ }^{2}$, M Nigou', C Auzan ${ }^{2}$, M O North ${ }^{1}$, J Sa ${ }^{6}$, L Gomes ${ }^{5}$, P Salpea ${ }^{8}$, \\ A Horvath $^{8}$, C A Stratakis ${ }^{8}$, N Hamzaoui ${ }^{1}$, J Bertherat ${ }^{3,4}$ and E Clauser ${ }^{1,2}$ \\ ${ }^{1}$ Département de Biologie Hormonale, Hôpital Cochin, Assistance Publique - Hôpitaux de Paris, 27 Rue du Faubourg \\ Saint Jacques, 75014 Paris, France, ${ }^{2}$ INSERM U970, Université Paris Descartes, PARCC, 56 Rue Leblanc, 75015 Paris, \\ France, 3'Service d'Endocrinologie, Hôpital Cochin, Assistance Publique - Hôpitaux de Paris, 75014 Paris, France, \\ ${ }^{4}$ INSERM U1060, CNRS, Institut Cochin, Université Paris Descartes, Paris, France, ${ }^{5}$ Serviço de Endocrinologia \\ ${ }^{6}$ Serviço de Genetica Medica, Departamento Pediatrico, Centro Hospitalar e Universitário de Coimbra, Coimbra, \\ Portugal, ${ }^{7}$ Clinical and Molecular Genetics Unit, UCL Institute of Child Health, London, UK and ${ }^{8}$ Section on \\ Endocrinology and Genetics, Eunice Kennedy Shriver National Institute of Child Health and Human Development \\ (NICHD), National Institutes of Health (NIH), Bethesda, Maryland 20892, USA \\ *(M Guillaud Bataille and Y Rhayem contributed equally to this work)
}

Correspondence

should be addressed to

E Clauser at INSERM U970,

Université Paris Descartes,

PARCC

Email

eric.clauser@inserm.fr

\begin{abstract}
Background: Point mutations of the PRKAR1A gene are a genetic cause of Carney complex (CNC) and primary pigmented nodular adrenocortical disease (PPNAD), but in $30 \%$ of the patients no mutation is detected.

Objective: Set up a routine-based technique for systematic detection of large deletions or duplications of this gene and functionally characterize these mutations.

Methods: Multiplex ligation-dependent probe amplification (MLPA) of the 12 exons of the PRKAR1A gene was validated and used to detect large rearrangements in 13 typical CNC and 39 confirmed or putative PPNAD without any mutations of the gene. An in-frame deletion was characterized by western blot and bioluminescence resonant energy transfer technique for its interaction with the catalytic subunit.

Results: MLPA allowed identification of exons 3-6 deletion in three patients of a family with typical CNC. The truncated protein is expressed, but rapidly degraded, and does not interact with the protein kinase A catalytic subunit.

Conclusions: MLPA is a powerful technique that may be used following the lack of mutations detected by direct sequencing in patients with bona fide CNC or PPNAD. We report here one such new deletion, as an example. However, these gene defects are not a frequent cause of CNC or PPNAD.
\end{abstract}

\section{Introduction}

Inactivating mutations of the type 1a regulatory subunit of cAMP-dependent protein kinase A gene (PRKAR1A) cause an autosomal dominant disorder, Carney complex (CNC) $(1,2)$. This syndrome is a multiple endocrine neoplasia syndrome associated not only with primary pigmented nodular adrenocortical disease (PPNAD), pituitary, thyroid, and ovarian tumors but also with cardiac and cutaneous myxomas and lentiginosis (3). PRKAR1A mutations are found in $\sim 60 \%$ of the cases of CNC. Occasional mutations led to apparently isolated (c) 2014 European Society of Endocrinology Printed in Great Britain
Published by Bioscientifica Ltd. 
PPNAD $(4,5)$. The frequency of PRKAR1A mutations in sporadic PPNADs is not known but appears to be lower than that in classical CNC.

More than 120 different mutations of the PRKAR1A gene have been identified so far (recently reviewed in $(6,7))$ and most are frameshift, splice site, or nonsense mutations (84\%) resulting in a rapid degradation of the mutated mRNA by nonsense-mediated mRNA decay.

It is intriguing that less than three-quarters of patients with classic $\mathrm{CNC}$, and even fewer of those with sporadic PPNAD, have a mutation in the PRKAR1A gene. This may suggest that other genes are involved, such as the recently identified PDE 8 and PDE11 genes $(8,9)$. But, there is also the possibility that some other sequence defects of the gene are not identified by direct sequencing, such as large rearrangements of the gene. Only three large deletions of the gene have been identified so far $(10,11)$, but in the absence of a systematic approach to search for them, their true frequency in CNC or PPNAD is unknown.

In this study, a routine-based technique was developed for the detection of large deletions or duplications of the PRKAR1A gene using multiplex ligation-dependent probe amplification (MLPA). This technique was applied to a series of patients with CNC and PPNAD, who were negative for any PRKAR1A mutations by Sanger sequencing. A large in-frame deletion of exons 3-6 was identified in a family with CNC and the expressed deletion mutant was structurally and functionally characterized.

\section{Subjects and methods}

\section{Patients}

The PRKAR1A gene sequence was analyzed in 46 cases with CNC and 53 with isolated PPNAD in our oncogenetic laboratory from January 2003 to June 2011. The diagnosis of CNC was made according to standard criteria (12). Patients with isolated myxoma or lentiginosis were not included in the study.

The diagnosis of PPNAD was made on the basis of either histological observations after adrenalectomy or association of an ACTH-independent Cushing's syndrome with normal or micronodular aspect of the adrenal glands upon imaging with bilateral adrenal uptake of iodocholesterol scintigraphy when this examination was performed. Patients with suspected bilateral adrenal macronodular hyperplasia or unilateral tumors were excluded from the study.

\section{MLPA of the PRKAR1A gene}

In the absence of a commercially available kit for the PRKAR1A gene, a homemade kit was developed for the 12 exons of this gene and contained the classical reagents for the MLPA reaction provided by MRC Holland (Amsterdam, The Netherlands) and 12 MLPA probes, each corresponding to one exon and composed of two oligonucleotides (Table 1a). Owing to the presence of a pseudogene, which presents $89 \%$ homology with the coding sequence of hPRKAR1A (13), most of the designed probes overlapped the adjacent intronic sequences. These probes were strictly exonic for exons $3,4,7,9$, and 11 . Two different MLPA reactions were set up due to the size limitation of the probes. MLPA mix A contains probes for exons 1a, 2, 4, 6, 8, and 10 and four control probes within the reference genes (Table 1c). MLPA mix B contains probes for exons $1 b, 3,5,7,9$, and 11 and for the same four control probes. For each MLPA reaction, $0.1 \mathrm{nM}$ of each oligonucleotide targeting PRKAR1A exons and references of either MLPA mix A or mix B were mixed with 250 to $500 \mathrm{ng}$ of genomic DNA. Hybridization of the primers on genomic DNA was carried out for $16 \mathrm{~h}$ at $60{ }^{\circ} \mathrm{C}$ after the denaturation of genomic DNA $\left(98^{\circ} \mathrm{C}\right.$ for $\left.5 \mathrm{~min}\right)$. The ligation and PCR steps were performed according to MRC Holland standard protocols using the EK-kit containing buffers, ligase, polymerase, and primers necessary for ligation and PCRs. The ligation step $\left(54^{\circ} \mathrm{C}\right.$ for $\left.15 \mathrm{~min}\right)$ and 35 cycles of PCR $\left(95^{\circ} \mathrm{C}-30 \mathrm{~s}, 60^{\circ} \mathrm{C}-30 \mathrm{~s}\right.$, and $72{ }^{\circ} \mathrm{C}-1 \mathrm{~min}$ ) were processed in a GeneAmp PCR system (Applied Biosystems). The fragments were then separated on a Beckman CEQ 8000 DNA sequencer (Beckman Coulter Genomics, Danvers, MA, USA). Specific peaks, corresponding to each exon, were identified according to their migration relative to the molecular size standards and results were exported to a Microsoft Excel spreadsheet. First, peak intensity was normalized by slope correction according to a linear regression between the sizes and intensities of the peaks of the reference probes, in order to remove the contribution of probe size to its peak intensity. Second, assessment of copy number alterations was performed with the REX-MLPA (regression-enhanced MLPA) method. REX-MLPA is based on an iterative regression between case and control samples (three control samples were included in each experiment). Starting with control probes, the regression defines the threshold for which target probes are considered to have no change in copy number. Such probes are used to re-estimate the thresholds. Test probes that fall outside the confidence intervals are classified as having either a gain or a loss in 
Table 1 Pairs of oligonucleotides synthesized for the MLPA technique. In all cases, universal PCR primer tags are added at $5^{\prime}$-end of LHS (5'-GGGTTCCCTAAGGGTTGGA-3') and at $3^{\prime}$-end of RHS (5'-TCTAGATTGGATCTTGCTGGCAC-3').

\begin{tabular}{|c|c|c|c|}
\hline & \multicolumn{2}{|c|}{ Oligonucleotide sequences } & \multirow{2}{*}{$\begin{array}{l}\text { Size } \\
\text { (bp) }\end{array}$} \\
\hline & Left hybridization sequence & Right hybridization sequence & \\
\hline \multicolumn{4}{|c|}{ a) PRKAR1A gene ${ }^{a}$} \\
\hline EXON 1A & $5^{\prime}$-GGAAAGGAGGGAGAAAAGGCAGAGGCGTC-3' & 5'-AAGGGAGGCCGGAGGGAGAGTGGGGTGGA-3' & 100 \\
\hline EXON 1B & 5'-GTTTCCGGTGGAGCTGTCGCCTAGCCGCT-3' & 5'-ATCGCAGAGTGGAGCGGGGCTGGGAGCAA-3' & 100 \\
\hline EXON 2 & 5'-CAG GGAATACTTT GAGAGGTTGG AGAAGGTAAA-3' & 5'-AATAAATGTGGGGAGATGATGAGGTGATTGTGA-3' & 108 \\
\hline EXON 3 & $5^{\prime}$-CCAGTGGTTAAAGGTAGGAGGCGACGAGGTGCT-3' & 5'-ATCAGCGCTGAGGTCTACACGGAGGAAGATGCG-3' & 108 \\
\hline EXON 4 & 5'-CTAGGTTATACCAAAAGATTACAAGACAATGGCCGCT-3' & 5'-TTAGCCAAAGCCATTGAAAAGAATGTGCTGTTTTCAC-3' & 116 \\
\hline EXON 5 & $\begin{array}{l}\text { 5'-TGCCATGTTTTCGGTCTCCTTTATCGCAGGAGAGACTGT- } \\
\text { GATTC-3' }\end{array}$ & 5'-AGCAAGGTAAGGGCCTCTGGAGCATGCAAT-3' & 116 \\
\hline EXON 6 & $\begin{array}{l}\text { 5'-CTCTTTTAGGTGATGAAGGGGATAACTTCTATGTGATT- } \\
\text { GAT-3' }\end{array}$ & $\begin{array}{l}\text { 5'-CAAGGAGAGACGGATGTAAGATTTACCAATATCAAAAA- } \\
\text { TAT-3' }\end{array}$ & 124 \\
\hline EXON 7 & $\begin{array}{l}\text { 5'-GATTTATGGAACACCGAGAGCAGCCACTGTCAAAG- } \\
\text { CAAAGA-3' }\end{array}$ & $\begin{array}{l}\text { 5'-CAAATGTGAAATTGTGGGGCATCGACCGAGACAGCTA- } \\
\text { TAGA-3' }\end{array}$ & 124 \\
\hline EXON 8 & $\begin{array}{l}\text { 5'-CGGAAGATGTATGAGGAATTCCTTAGTAAAGTCTCTATTT- } \\
\text { TAGG T-3' }\end{array}$ & $\begin{array}{l}\text { 5'-GAGTTGTAAAGTGTGTTAACTTTGCTAGTATGTGAGA- } \\
\text { TACCCCTG-3' }\end{array}$ & 132 \\
\hline EXON 9 & $\begin{array}{l}\text { 5'-GTCTTACGGTAGCTGATGCATTGGAACCAGTGCAGTTT- } \\
\text { GAAGATG-3' }\end{array}$ & $\begin{array}{l}\text { 5'-GGCAGAAGATTGTGGTGCAGGGAGAACCAGGGGAT- } \\
\text { GAGTTCTTCA-3' }\end{array}$ & 132 \\
\hline EXON 10 & $\begin{array}{l}\text { 5'-TTGGTGATTTTATTATAGGGGGTCAGCTGCTGTGCTA- } \\
\text { CAACGTCGGTC-3' }\end{array}$ & $\begin{array}{l}\text { 5'-AGAAAATGAAGAGTTTGTTGAAGTGGGAA- } \\
\text { GATTGGGGCCTTCTGATT-3' }\end{array}$ & 136 \\
\hline EXON 11 & $\begin{array}{l}\text { 5'-G TGCGTTAAGCTGGACCGACCTAGATTT- } \\
\text { GAACGTGTTCTTGGCCCAT-3' }\end{array}$ & $\begin{array}{l}\text { 5'-GCTCAGACATCCTCAAACGAAACATCCAGCAGTACAA- } \\
\text { CAGTTTTGTG-3' }\end{array}$ & 136 \\
\hline \multicolumn{4}{|c|}{ b) MLPA probes ${ }^{\mathrm{b}}$} \\
\hline EXON 3 & 5'-GGTTAAAGGTAGGAGGCGACGAGGTGCTA-3' & 5'-TCAGCGCTGAGGTCTACACGGAGGAAGAT-3' & 100 \\
\hline IVS2_B & 5'-TTCCTGGAGATCCGGTTTTGAAATGGGTCAC-3' & 5'-TGTAAGGTGATCCCTCTTCTTTGGACATCAA- $3^{\prime}$ & 104 \\
\hline IVS2_C & 5'-GGGGGGTTGTGTTTCTGTTACCTTCAACTACTCCA-3' & 5'-TAAGAAATGTACCAAAAACTCTTGACAGTTGGTCT-3' & 112 \\
\hline IVS2_D & 5'-GCTCTGAGTTGTCAGATTAGGTTATATTCAGATTGCT-3' & 5'-GTTTTTAAAAGACTCACTAGAATCCATGAGACACTGC-3' & 116 \\
\hline IVS2_E & $\begin{array}{l}\text { 5'-CGGGTCAAATGAATGAGAAAGTAGAAGATAACA- } \\
\text { CATTTTT-3' }\end{array}$ & 5'-TATTTTTTTCCCTTTTACTTCAGGCACAACAACTAATTCT-3' & 120 \\
\hline IVS6 & $\begin{array}{l}\text { 5'-GTTCTCTAAGTATCTGTGACTGGTATTAGTGCTTAGCT- } \\
\text { GACCT-3' }\end{array}$ & $\begin{array}{l}\text { 5'-TTTTCCCTTACATCCTTACAGAGTGCTTTTTTCTTATCA- } \\
\text { GATA-3' }\end{array}$ & 128 \\
\hline \multicolumn{4}{|c|}{ c) Reference genes ${ }^{c}$} \\
\hline SERPINB2 & 5'-CAGAGAACTTTACCAGCTGTGGGTTCATGCA-3' & 5'-GCAGATCCAGAAGGGTAGTTATCCTGATGCG-3' & 104 \\
\hline$D A C H 1$ & 5'-CTAGACCTGGAAGGCCTCCTAAGAGGACTCAAA-3' & 5'-GTGTCACCTCCCCAGAGAACTCTCACATCATGCCGCA-3' & 112 \\
\hline ING1 & $\begin{array}{l}\text { 5'-CAGAGATCCTGAAGGAGCTAGACGAGTGCTAC- } \\
\text { GAGCGCT-3' }\end{array}$ & $\begin{array}{l}\text { 5'-TCAGTCGCGAGACAGACGGGGCGCAGAAGCGGCG- } \\
\text { GATGC-3' }\end{array}$ & 120 \\
\hline SS18 & $\begin{array}{l}\text { 5'-GACAGCATTACCAAGGACAGCAGCCACCTATGGGAAT- } \\
\text { GATG-3' }\end{array}$ & $\begin{array}{l}\text { 5'-GGTCAAGTTAACCAAGGCAATCATATGATGGGTCAGAGA- } \\
\text { CAGATT-3' }\end{array}$ & 128 \\
\hline
\end{tabular}

asequences of the 12 pairs of oligonucleotides designed to analyze the 12 exons of PRKAR1A gene.

${ }^{\mathrm{b}}$ Sequences of the oligonucleotides designed specifically to localize the breakpoints of exons 3-6 deletion in one family of CNC.

'Sequences of the oligonucleotides designed for the 4 reference genes.

copy number $(14,15)$. The spreadsheet templates used for the data analysis reported here are available upon request.

In order to validate the method, series of normal genomic DNA and a set of plasmids containing the PRKAR1A cDNA with or without deletions of exons 3, 4, 7,9 , or 11 were analyzed. As indicated in Supplementary Figure 1, see section on supplementary data given at the end of this article, the exonic deletion was identified for each of these plasmids, in comparison with the WT plasmid.

An additional MLPA assay, using the same protocol described above, was set up specifically for introns 3 and 6 in order to identify the breakpoint regions in one CNC family suspected to present a large deletion (see below). The oligonucleotides used for the MLPA probes are indicated in Table $1 \mathrm{~b}$.

\section{PRKAR1A cDNA constructs}

The coding sequence for human PRKAR1A gene was obtained by RT-PCR and subcloned as previously described (16). To establish controls for PRKAR1A MLPA, each of the five exons (exons 3, 4, 7, 9, and 11) presenting a purely exonic MLPA probe was deleted in the cDNA sequence by site-directed mutagenesis using the Quick change Site-directed mutagenesis kit (Stratagene, LaJolla, 
Table 2 Clinical and mutational summaries of groups of familial and sporadic CNC.

\begin{tabular}{|c|c|c|c|c|c|c|c|c|c|c|}
\hline & $n$ & Age & $\operatorname{Sex}(M / F)$ & PPNAD & Myxoma & Lentiginosis & $\begin{array}{l}\text { Other } \\
\text { tumors }\end{array}$ & $\begin{array}{l}\text { PRKAR1A } \\
\text { mutation }\end{array}$ & $\begin{array}{c}\text { PRKAR1A } \\
\text { deletion }\end{array}$ & $\begin{array}{c}\text { PDE11A } \\
\text { mutation }\end{array}$ \\
\hline Familial CNC & 19 & $37 \pm 18$ & $8 / 11$ & 10 & 11 & 16 & 8 & 14 & 1 & 0 \\
\hline Sporadic CNC & 27 & $40 \pm 17$ & $7 / 20$ & 18 & 18 & 20 & 7 & 19 & 0 & 1 \\
\hline
\end{tabular}

CA, USA). The oligonucleotides used for mutagenesis are described in Supplementary Table 1, see section on supplementary data given at the end of this article.

The deletion mutant observed in one family of CNC was reproduced by site-directed mutagenesis with the oligonucleotides described in Supplementary Table 1. As previously described (16), two plasmids were used as templates: pcDNA3-hPRKAR1A and phPRKAR1A-hRluc. The resulting plasmids were called phPRKAR1A- $\Delta \mathrm{E} 3-6$ and phPRKAR1A-hRluc- $\Delta$ E3-6 respectively.

\section{Functional characterization of the deletion mutant}

EBV-immortalized B-lymphocytes of one of the CNC patients (II-2) with a deletion of PRKAR1A gene and HEK293 transfected with the phPRKAR1A- $\Delta$ E3-6 or the WT phPRKAR1A constructs were solubilized and analyzed by western blot using classical procedures. The PRKAR1A proteins were identified with the antiPKA (R1) antibody (\#610166, BD Transduction Laboratories, NJ, USA). The degradation of the WT and mutated PRKAR1A proteins was characterized on the same transfected HEK293 cells after treatment with cycloheximide $(20 \mu \mathrm{g} / \mathrm{ml})$ for $0-4 \mathrm{~h}$, followed by western blot.

Interactions between regulatory and catalytic subunits of the PRKAR1A proteins were analyzed by bioluminescence resonance energy transfer (BRET) assay, as previously described $(16,17)$.

\section{Results}

\section{CNC, PPNAD, and PRKAR1A gene mutations}

Between January 2003 and June 2011, 99 kindred were investigated for a loss-of-function mutation of the
PRKAR1A gene (Tables 2 and 3). This genetic test was indicated for a suspicion of $\mathrm{CNC}$ (46 cases) or isolated PPNAD (53 cases). There were 19 familial cases of CNC. A PRKAR1A point mutation was identified in 33 of the 46 analyzed CNC cases (77\%). In 13 cases, no mutation was identified by sequencing. The diagnosis of isolated PPNAD was suspected in 53 cases presenting either hypercortisolism with histologically confirmed PPNAD (39/53 cases) or ACTH-independent hypercortisolism with normal or bilateral micronodular aspect of the adrenal glands at magnetic resonance imaging and/or bilateral hyperfixation of the adrenal at iodocholesterol scintigraphy (14/53 cases). A mutation of PRKAR1A was identified in 14 cases (26\%), 13 of them in the group of histological PPNADs (33\%) and one of them in the group of clinical PPNADs (7\%). Therefore, more than half of the genetic investigations (52/99) were unsuccessful for the PRKAR1A gene and one possible explanation was that large rearrangements of the gene are missed by sequencing.

\section{PRKAR1A gene deletion in a family with CNC}

Using MLPA assay, the DNA of 13 CNC and 32 PPNAD patients, presenting no identified point-mutation by sequencing, were analyzed for exonic insertion/deletion of the PRKAR1A gene. None of the PPNAD patients exhibited a potential large rearrangement of the gene, and only one sample in the patients with CNC was strongly suspected to exhibit a deletion of the gene.

Pedigree of this family is shown in Fig. 1, and clinical presentation of different relatives of this family is detailed in Table 4. The proband (I-2) belongs to a Portuguese family. He underwent two surgeries for cardiac myxoma, the first one at the age of 62 years. He died 2 years ago at 72 years old. His daughter (II-2) is 52 years old and presents

Table 3 Clinical and mutational summaries of groups of confirmed or suspected PPNAD. Details on the clinical definition of each group are described in the 'Subjects and methods' section.

\begin{tabular}{|c|c|c|c|c|c|c|}
\hline & $n$ & Age & $\operatorname{Sex}(M / F)$ & $\begin{array}{l}\text { PRKAR1A } \\
\text { mutation }\end{array}$ & $\begin{array}{c}\text { PRKAR1A } \\
\text { deletion }\end{array}$ & $\begin{array}{l}\text { PDE11A } \\
\text { mutation }\end{array}$ \\
\hline Confirmed PPNAD & 39 & $28 \pm 15$ & $5 / 33$ & 13 & 0 & 4 \\
\hline Suspected PPNAD & 14 & $34 \pm 17$ & $2 / 12$ & 1 & 0 & 1 \\
\hline
\end{tabular}




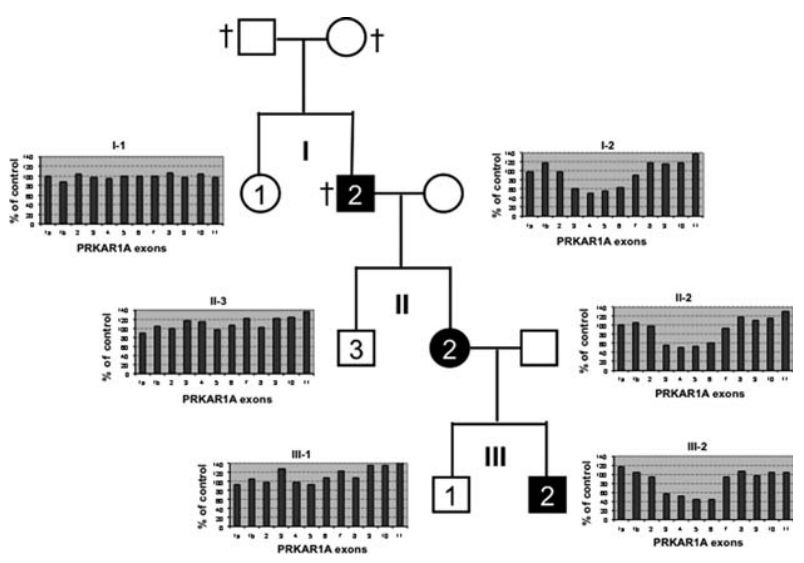

\section{Figure 1}

Genealogical tree of a Portuguese family analyzed for PRKAR1A gene deletion at three generations (labeled in roman numerical: I, II, and III). Three patients (I-2, II-2, and III-2) presented with a typical CNC and are represented as black squares (male) or circle (female) with number in white. Three siblings without any CNC clinical signs were also studied (I-1, II-3, and III-1, white squares or circle with black numbers). Deceased persons are indicated by $t$. MLPA results for each of the studied patients are represented as a box containing a histogram ( $x$-axis, number of the studied exon; $y$-axis, value $(\%)$ of the area of the peak compared with a panel of controls). A value of $100 \%$ indicates the presence of two alleles: $50 \%$ one allele and $0 \%$ the absence of exon detection.

with a typical clinical profile of CNC. She underwent several surgeries: bilateral adrenalectomy for PPNAD at age 10, resection of a GH-producing pituitary adenoma in early adulthood, followed by a cardiac myxoma surgery at age 31 and resection of breast and uterine adenoma. Her son (III-2) is 19 years old and underwent cardiac surgery for myxoma at 7 months of age followed by a unilateral adrenalectomy for PPNAD at the age of 7 and a dorsal myxoma surgery at the age of 17 years (Table 4). All three first-degree relatives were presented with multiple skin tags, suggesting lentiginosis. Other studied members of the family had no symptom of $\mathrm{CNC}$, including individuals I-1, II-3, and III-1 of the pedigree (Fig. 1).

Genomic DNA of patients I-2 and II-2 was extensively sequenced for the identification of point-mutation in the coding sequence of the PRKAR1A gene. No mutation was observed in the 12 exons of the PRKAR1A gene. As for the other studied cases of typical CNC or PPNAD with no identified mutation, the PRKAR1A gene was investigated for large rearrangements. As shown in Fig. 1, the DNA of the two patients (I-2 and II-2) presented a deletion of exons
3-6. Four other members of the family were investigated for this deletion and only patient III-2, but not relatives I-1, II-3, and III-1, presented the same deletion. This result was confirmed on a second independent DNA sample for patients I-2, II-2, and III-2.

In order to confirm this deletion and identify its breakpoints, we performed several strategies. We first designed primers every $500 \mathrm{bp}$ across the $10 \mathrm{~kb}$ sequence between the end of exon 2 and the beginning of exon 7 to amplify the fragments to be sequenced (data not shown). Unfortunately, no PCR fragment was identified. Second, we analyzed the $10 \mathrm{~kb}$ sequence between exons 2 and 7 and identified two Alu sequences at the end of PRKAR1A intron 2 and one in intron 6, with respectively 67 and $77 \%$ identity with the two intron 2 Alu sequences. This suggested a potential recombination event between two of these sequences. Oligonucleotides designed before the first (intron 2) Alu sequence and after the last (intron 6) Alu sequence failed to amplify a PCR fragment in the CNC patient II-2. Finally, we designed five MLPA probes (Table $1 \mathrm{~b}$ ) in the $10 \mathrm{~kb}$ potentially deleted for the patients in order to narrow the search of the breakpoints (Fig. 2A and $\mathrm{B})$. The four MLPA probes located in intron 2 gave a deletion profile, indicating that intron 2 was almost totally deleted. However, the MLPA probe located in intron 6 had a normal profile, whereas a second probe to exon 6 confirmed its deletion. Altogether, these data approximately located the breakpoints and allowed us to design appropriate oligonucleotides to amplify and sequence the affected region. Figure $2 \mathrm{C}$ shows the chromatogram of the sequence around the breakpoints. The $5^{\prime}$ breakpoint is at position 66512656 on chromosome 17 and the $3^{\prime}$ breakpoint is at position 66521 194, resulting in a deletion of $8538 \mathrm{bp}$. No homology between the two regions around the $5^{\prime}$ and $3^{\prime}$ breakpoints was identified, with the exception of a short $5 \mathrm{bp}$ sequence (TCATT). This analysis confirms the results observed by MLPA and validates this assay to identify large rearrangements of the PRKAR1A gene.

\section{Functional consequences of exons 3-6 deletion}

Deletion of exons 3-6 should correspond to a shorter mRNA and a truncated protein if expressed, resulting in haploinsufficiency. A careful analysis of the deleted sequence indicates that deletion of exons 3-6 was an in-frame deletion, potentially resulting in a new protein missing aminoacids 60-183, corresponding to the four last aminoacids of the dimerization domain, the inhibitory site, which interacts with the catalytic subunit and is the beginning of the cAMP-binding domain A (Fig. 3). 
Table 4 Clinical presentation of a family with Carney complex (CNC). Three individuals with CNC and three individuals without any clinical symptom of CNC were analyzed for point mutation and large rearrangement of the PRKAR1A gene.

\begin{tabular}{|c|c|c|}
\hline Patients & Sex (M/F) & Age (years) \\
\hline I-1 & $\mathrm{F}$ & 71 \\
\hline $1-2$ & $M$ & 72 \\
\hline
\end{tabular}

II-2

II-3

III-1

III-2

\section{Adrenal disease}

(age at diagnosis)

$-$

$-$

ACTH independent Cushing's syndrome

Bilateral adrenalectomy (10)

PPNAD

Unilateral adrenalectomy (7)

\section{Myxoma \\ (age at diagnosis) \\ Cardiac myxoma (62) \\ Second cardiac \\ myxoma - surgery \\ Cardiac myxoma (31) \\ Multiple cutaneous myxoma \\ Cardiac myxoma \\ (30 months)}

\begin{tabular}{c}
\hline Lentiginosis \\
\hline $\begin{array}{c}\text { Yes } \\
\text { Yes }\end{array}$ \\
\\
- \\
- \\
Yes
\end{tabular}

Dorsal myxoma, surgery at 17 years old, probable GH pituitary adenoma currently under evaluation
This truncated protein was analyzed by western blot in HEK293 cells transfected with the mutated cDNA or in lymphocytes of patient II-2. As shown in Fig. 4A, the truncated PRKAR1A protein is expressed as a $31 \mathrm{kDa}$ band, in addition to the WT protein $(49 \mathrm{kDa})$ expressed by the other allele (patient lymphocytes) or the endogenous PRKAR1A gene of HEK293. The intensity of the band for the truncated protein was weaker than that of the WT protein expressed in the lymphocytes, or equal to the endogenous protein expressed in HEK293 despite overexpression of the mutated one. This observation suggests a lower expression of the truncated protein either by reduction of its biosynthesis or by increase of its degradation. We further investigate the latter hypothesis by measuring the half lives of the WT and mutated proteins after blocking their biosynthesis with cycloheximide (Fig. 4B). As shown on the quantification in Fig. 4C, the half-life of the truncated protein is very short $(<2 \mathrm{~h})$, whereas that of the WT protein is between 4 and $5 \mathrm{~h}$.

Since the degradation of PRKAR1A is dependent on its association to the catalytic subunit, we investigated whether this truncated regulatory subunit is still able to bind to the catalytic subunit. The deletion of the interacting site and the rapid degradation of the truncated protein suggested an abnormal binding to the catalytic subunit. This was confirmed experimentally using BRET: with increasing concentrations of PRKAC1A-YFP, no BRET signal was detected for $\Delta 1$-6-PRKAR1A-luciferase, whereas a strong and saturable signal was observed for the WT PRKAR1A-luciferase (Fig. 5).

Altogether, these data indicate that CNC in the three patients of this Portuguese family is the consequence of a deletion of exons 3-6 of the PRKAR1A gene. This gene deletion results in the transcription and translation of a truncated protein with a shorter half-life probably in part due to its absence of association with the catalytic subunit. The resulting consequence remains haploinsufficiency.

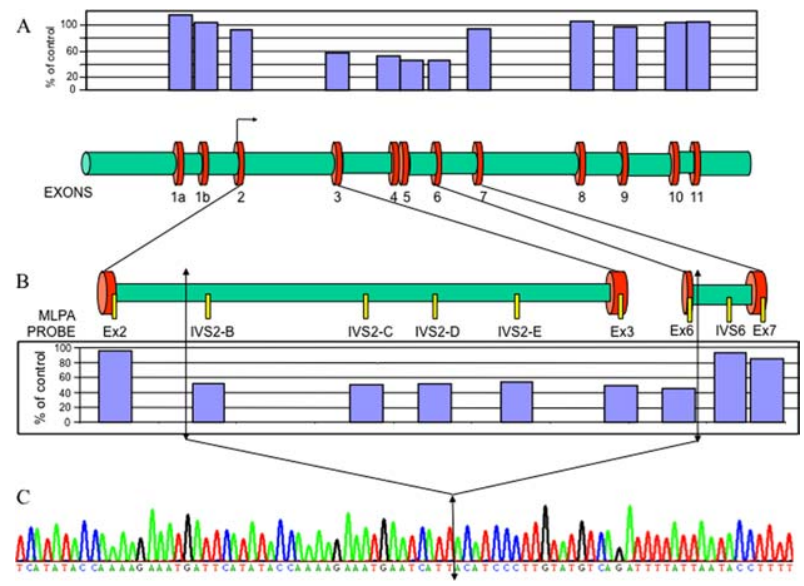

\section{Figure 2}

Breakpoints localization of the deletion of exons 3-6. (A, lower part) Structure of the PRKAR1A gene at scale with the 12 exons and the introns. (A, upper part) MLPA histogram for patient II-2 showing a heterozygous deletion of exons 3-6. The breakpoints are therefore located in introns 2 and 6. (B, upper part) Schematic representation of introns 2 and 6 with the positions of the different MLPA probes designed to locate the breakpoints. (B, lower part) Histograms of this specific MLPA analysis identify the breakpoints at the proximal sequences of introns 2 and 6. Arrows locate these potential breakpoints. (C) Sequence analysis around the breakpoints, identifying precisely the size of the deletion. The double arrow indicates the fusion point between introns 2 and 6 . 


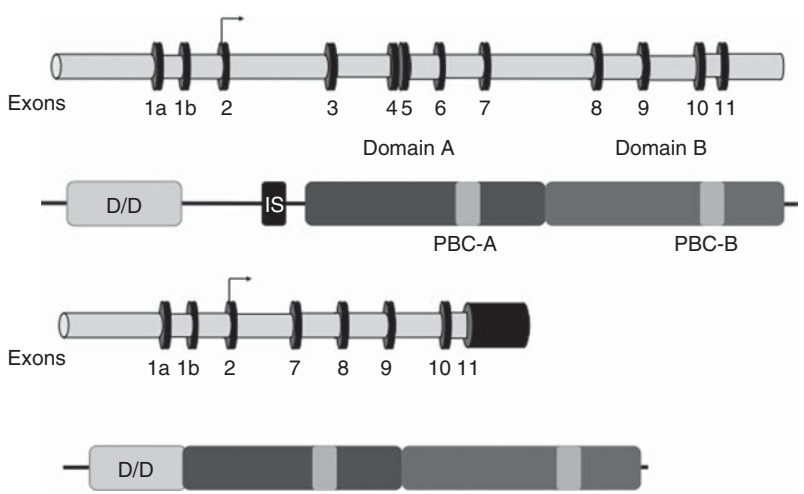

Figure 3

Schematic representation of the WT and $\Delta$ exons 3-6 mutant PRKAR1A genes and proteins. The genes are represented with exonic sequences (black and numbered) and intronic sequences (grey). The proteins are represented with their functional domains: dimerization domain (D/D); inhibitory sequence which binds to the catalytic subunit (IS); and the two CAMP-binding domains ( $A$ in dark grey and $B$ in light grey) with their phosphobinding cassettes (PBC). The deleted mutant protein lacks the last four amino acids of the dimerization domain, the inhibitory sequence and the first 41 aminoacids of cAMP-binding domain A.

\section{Discussion}

In this study, large rearrangements of $P R K A R 1 A$ gene were investigated in CNC and sporadic PPNAD using a MLPA technique. For 8 years, our laboratory was able to analyze the PRKAR1A sequence in order to detect point mutations in 46 kindred of CNC and 53 cases of sporadic PPNAD. The CNC kindred were selected for their typical clinical presentation, i.e. familial cases of CNC (19 kindred) with at least two of the three cardinal lesions (PPNAD, myxoma, and lentiginosis) associated or not with other tumors found classically in CNC. Sporadic cases of CNC (27 kindreds) met the same criteria of diagnosis, but such a diagnosis was identified in only one member of the kindred at the time of the genetic test. Considering the clinical information obtained by the oncogenetic laboratory at the time of the genetic test, the observed ratio between familial vs sporadic CNC (40 vs 60\%) is unusual compared with the ratio reported in the literature (70 vs $30 \%$ ) from the clinician point of view (6). Our cohort excluded isolated myxoma or lentiginosis. As already described, probands of CNC are more often women $(67 \%)$ than men (33\%) and the mean age at diagnosis is around 40 years old (6). In this selected population, high frequencies of PPNAD (61\%), myxoma (63\%), and lentiginosis (78\%) were observed. As expected, the frequency of $P R K A R 1 A$ point mutation in this classical CNC cohort is high (72\%). It is higher than the $62 \%$ described recently in a cohort of 185 families of $\mathrm{CNC}$ and/or PPNAD (6).

Isolated PPNAD is another clinical situation, for which investigation of the PRKAR1A sequence is necessary (5). In this study, we have distinguished the histologically confirmed PPNAD from the PPNAD suspected on clinical and imaging criteria. We confirm previous studies indicating that PRKAR1A gene point mutations are frequent in confirmed isolated PPNAD (33\%), but clearly less frequent than in classical CNC. Interestingly, the frequency of identified mutations is very low (7\%) in PPNAD suspected on fluctuant ACTH-independent

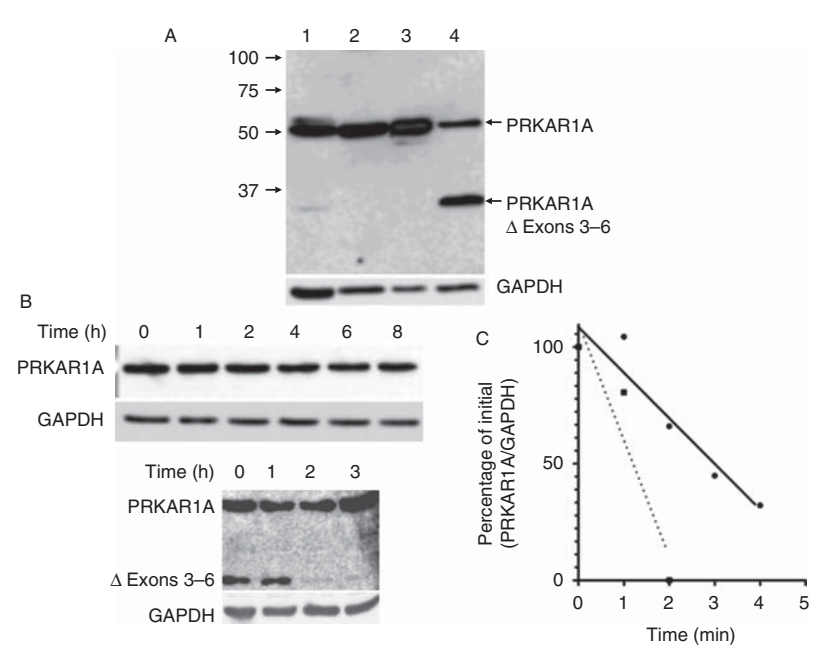

\section{Figure 4}

Expression and degradation of the WT and $\Delta 3-6$ deletion mutant. (A) Western blot analysis of PRKAR1A. Lane 1, lymphocytes of patient II-2 (60 $\mu$ g protein extract); lane 2, HEK 293 cells $(60 \mu \mathrm{g}$ protein extract); lane 3 , HEK 293 cells transfected with the WT PRKAR1A construct $(10 \mu \mathrm{g}$ protein extract); and lane 4, HEK293 cells transfected with the deletion mutant construct ( $20 \mu \mathrm{g}$ protein extract). Western blot of the same membrane for GAPDH is shown in the lower part of the figure as control. (B) Degradation of PRKAR1A in transfected cells. Western blot of PRKAR1A and GAPDH was performed on HEK293 cells transfected with WT (upper part) or deletion mutant (lower part) PRKAR1A constructs and after incubation with cycloheximide $(20 \mu \mathrm{g} / \mathrm{ml})$ for the indicated time. (C) Typical degradation curves for WT (black dots and solid line) or deletion mutant (black squares and dotted line). 


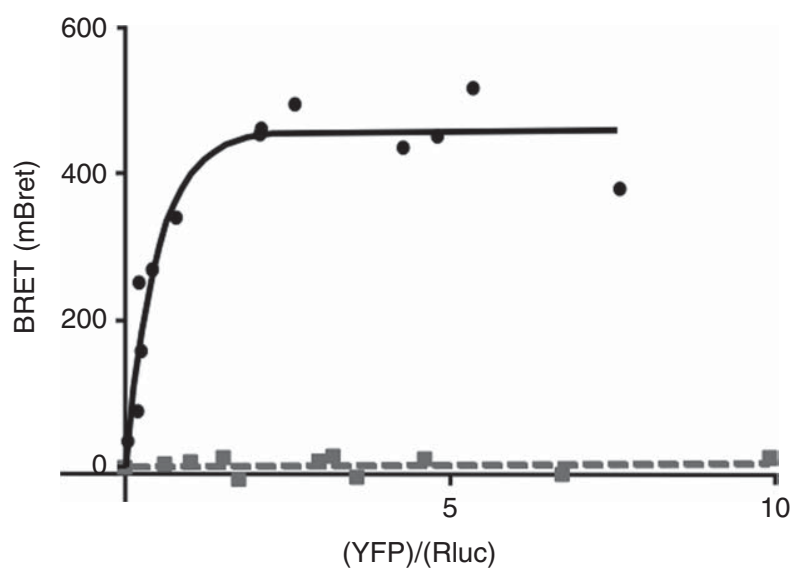

Figure 5

Interaction of the WT and truncated mutant PRKAR1A with the catalytic PKA subunit analyzed with BRET technique. Fusion proteins were designed corresponding to WT and deletion mutant of PRKAR1A-R-Luciferase and EYFP-PRKAC1A. These constructions were expressed in HEK293 cells with a constant expression of the luciferase constructs and increasing expression of the EYFP construct. BRET was measured for each condition and expressed as the arbitrary unit (MBRET; ordinate of the graph). In abscissa is represented as the ratio between the amounts of EYFP fusion protein and of the R-luciferase fusion protein. For the WT PRKAR1A (black dots and solid line) there is a saturable and important interaction between the two subunits, whereas the deletion mutant (grey squares and dotted line) is unable to interact with the catalytic subunit.

Cushing's syndrome with no alteration of the adrenal CT-scan and/or bilateral fixation at iodocholesterol scintigraphy. This observation indicates that these clinical and imaging criteria are insufficient for the diagnosis of PPNAD, and in rare cases genetic testing may help with the diagnosis. Taken altogether, $30-40 \%$ of the index cases of $\mathrm{CNC}$ and at least $66 \%$ of the isolated PPNAD had no point mutation of the PRKAR1A gene, and investigation of other genetic causes (PDE11A and PDE8B mutations) for these remaining families estimated their frequency at $12 \%$ of the patients with PPNAD (7).

Another possibility never investigated on a large scale in absence of an appropriate methodology is the presence of large rearrangements of the PRKAR1A gene. The presented MLPA technique offers the possibility of such an investigation. In the $\mathrm{CNC}$ cohort presented in this study, there was a large rearrangement in one out of 13 families of $\mathrm{CNC}$ without any point mutation of the gene.
In addition, none of the 39 suspected or even confirmed PPNAD without PRKAR1A gene mutation presented a large rearrangement of the gene. From this study and a previous one (11), it is clear that the frequency of large deletions affecting the PRKAR1A gene is low. In the present cohort, it represents around $2 \%$ of the families, a number to compare with the $6 \%$ observed by Horvath et al. (11) in a series of $36 \mathrm{CNC}$ without small intragenic mutations.

The first reported large rearrangement of PRKAR1A gene corresponded to a complete deletion of not only this gene but also 13 adjacent genes, resulting in a severe phenotype of mental retardation and microcephaly in a young boy (10). Two other shorter deletions were described in apparently sporadic cases of CNC using Southern blot analysis (11). The first one is a $3876 \mathrm{bp}$ deletion including part of the promoter and the untranslated first exon of the PRKAR1A gene. This deletion should logically result in a quantitative defect in PRKAR1A expression and haploinsufficiency. The second is a $5149 \mathrm{bp}$ deletion including the third exon and parts of the flanking introns. Such a deletion should result in a shorter RNA. The present paper reports another deletion of 8538 bp (66 512 656-66 521194 bp) of chromosome 17 in the human genome sequence, which encompasses exons 3-6 and parts of the flanking introns. The recombination event resulting in this deletion did not involve the Alu sequences located in introns 2 and 6 , since the identified breakpoints are located either $4.5 \mathrm{~kb}$ above the exon $2 \mathrm{Alu}$ sequences or $130 \mathrm{bp}$ above the exon 6 homologous Alu sequence. The breakpoint includes a very short sequence of $5 \mathrm{bp}$ (TCATT) homologous in the two introns. Similar results were observed for the two other analyzed deletions (11). There is no doubt that systematic analysis of PRKAR1A gene large rearrangements using MLPA or other techniques in all CNC without PRKAR1A mutations will allow the identification of more CNC families with this type of alteration.

Finally, we found that the deletion of exons 3-6 results in an in-frame deletion with the potentially translated protein missing the last four aminoacids of the dimerization domain, the inhibitory sequence, and the first 41 aminoacids of cAMP-binding domain A. This observation is reminiscent of the previously reported deletion of exon 3 , which is also an in-frame deletion (11), and a splice site mutation $(c .708+1 \mathrm{G}>\mathrm{T})$ resulting in an exon 7 (former exon 6) skipping event (18). The deletion of exons 3-6 results in an expressed protein, which was detected both in the immortalized lymphocytes of the patients and in HEK293 cells transfected with the corresponding vector containing the mutant cDNA sequence. The apparent 


(1)

molecular weight (MW) was around $31 \mathrm{kDa}$, close to expected theoretical MW of this truncated protein $(29.15 \mathrm{kDa})$. This truncated protein is rapidly degraded as assessed by its half-life $<2 \mathrm{~h}$, leading to partial haploinsufficiency, whereas the WT protein has a longer half-life above $4 \mathrm{~h}$, as already described for the mouse PRKAR1A (19). The remaining undegraded truncated protein is unable to bind to the catalytic subunit as demonstrated by BRET techniques. Taken together, these functional alterations of PRKAR1A- $\Delta 3-6$ result in inappropriate activation of the enzyme and CNC phenotypes. Similar observations were made for the exons 3 and 7 deletion mutants described previously $(20,21) . \Delta 3$-PKAR1A mutant does not interact with the catalytic subunit and its overexpression results in increased activation of the PKA enzyme in response to cAMP. In addition, it presents a reduced binding to cAMP (20). The $\Delta 7$-PRKAR1A mutant results in a significantly higher cAMP-induced kinase activity compared with the WT, in both CRE-luciferase reporter assay and CREB phosphorylation $(5,18,21)$. Taken together, these data confirm that in-frame deletions of exon 3, exons 3-6, or exon 7 result in expressed proteins with defective properties of inhibitory binding to the catalytic subunits and therefore increased activity of the enzyme. Whether some of these deleted proteins are still able to form dimers is not known; however in this case, they may act as dominant negative by trapping the WT PRKAR1A subunits for example. Some of these dominant negative properties may explain the severe phenotype of CNC in these patients, which includes early onset of the disease, frequent occurrence of the three major localizations of the disease (PPNAD, myxoma, and lentiginosis), and also multiple other tumoral localizations. Interestingly, the presented family does not fit in this category, suggesting that the molecular consequences of the exons 3-6 deletion are not similar.

In conclusion, a routine-based assay by MLPA has been developed for the detection of PRKAR1A gene large rearrangements. This assay was validated and was used to detect such gene modifications in a series of CNC and PPNAD negative for a point-mutation of the PRKAR1A gene. This test was able to detect a deletion of exons 3-6 in a family of CNC, a diagnosis confirmed by direct sequencing of the breakpoints. The corresponding truncated protein is expressed, but is rapidly degraded and does not bind to the catalytic subunit.

\section{Supplementary data}

This is linked to the online version of the paper at http://dx.doi.org/10.1530/ EJE-13-0740.

\section{Declaration of interest}

The authors declare that there is no conflict of interest that could be perceived as prejudicing the impartiality of the research reported.

\section{Funding}

This work was supported by recurrent funding from INSERM and Assistance Publique - Hôpitaux de Paris.

\section{Acknowledgements}

Many thanks to Dr R Vargas Poussou for valuable discussions about Alu sequences.

\section{References}

1 Kirschner LS, Carney JA, Pack SD, Taymans SE, Giatzakis C, Cho YS, Cho-Chung YS \& Stratakis CA. Mutations of the gene encoding the protein kinase A type I- $\alpha$ regulatory subunit in patients with the Carney complex. Nature Genetics 200026 89-92. (doi:10.1038/79238)

2 Kirschner LS, Sandrini F, Monbo J, Lin JP, Carney JA \& Stratakis CA. Genetic heterogeneity and spectrum of mutations of the PRKAR1A gene in patients with the Carney complex. Human Molecular Genetics 20009 3037-3046. (doi:10.1093/hmg/9.20.3037)

3 Carney JA, Gordon H, Carpenter PC, Shenoy BV \& Go VL. The complex of myxomas, spotty pigmentation, and endocrine overactivity. Medicine 198564 270-283. (doi:10.1097/00005792-198507000-00007)

4 Groussin L, Horvath A, Jullian E, Boikos S, Rene-Corail F, Lefebvre H, Cephise-Velayoudom FL, Vantyghem MC, Chanson P, Conte-Devolx B et al. A PRKAR1A mutation associated with primary pigmented nodular adrenocortical disease in 12 kindreds. Journal of Clinical Endocrinology and Metabolism 200691 1943-1949. (doi:10.1210/ jc.2005-2708)

5 Groussin L, Jullian E, Perlemoine K, Louvel A, Leheup B, Luton JP, Bertagna X \& Bertherat J. Mutations of the PRKAR1A gene in Cushing's syndrome due to sporadic primary pigmented nodular adrenocortical disease. Journal of Clinical Endocrinology and Metabolism 200287 4324-4329. (doi:10.1210/jc.2002-020592)

6 Bertherat J, Horvath A, Groussin L, Grabar S, Boikos S, Cazabat L, Libe R, Rene-Corail F, Stergiopoulos S, Bourdeau I et al. Mutations in regulatory subunit type $1 \mathrm{~A}$ of cyclic adenosine 5 '-monophosphate-dependent protein kinase (PRKAR1A): phenotype analysis in 353 patients and 80 different genotypes. Journal of Clinical Endocrinology and Metabolism 200994 2085-2091. (doi:10.1210/jc.2008-2333)

7 Horvath A, Bertherat J, Groussin L, Guillaud-Bataille M, Tsang K, Cazabat L, Libe R, Remmers E, Rene-Corail F, Faucz FR et al. Mutations and polymorphisms in the gene encoding regulatory subunit type $1-\alpha$ of protein kinase A (PRKAR1A): an update. Human Mutation 201031 369-379. (doi:10.1002/humu.21178)

8 Horvath A, Boikos S, Giatzakis C, Robinson-White A, Groussin L, Griffin KJ, Stein E, Levine E, Delimpasi G, Hsiao HP et al. A genome-wide scan identifies mutations in the gene encoding phosphodiesterase 11A4 (PDE11A) in individuals with adrenocortical hyperplasia. Nature Genetics 200638 794-800. (doi:10.1038/ng1809)

9 Horvath A, Mericq V \& Stratakis CA. Mutation in PDE8B, a cyclic AMP-specific phosphodiesterase in adrenal hyperplasia. New England Journal of Medicine 2008358 750-752. (doi:10.1056/ NEJMc0706182)

10 Blyth M, Huang S, Maloney V, Crolla JA \& Karen Temple I. A 2.3Mb deletion of 17q24.2-q24.3 associated with 'Carney complex plus'. European Journal of Medical Genetics 200851 672-678. (doi:10.1016/ j.ejmg.2008.09.002) 
11 Horvath A, Bossis I, Giatzakis C, Levine E, Weinberg F, Meoli E, Robinson-White A, Siegel J, Soni P, Groussin L et al. Large deletions of the PRKAR1A gene in Carney complex. Clinical Cancer Research 2008 14 388-395. (doi:10.1158/1078-0432.CCR-07-1155)

12 Stratakis CA, Kirschner LS \& Carney JA. Clinical and molecular features of the Carney complex: diagnostic criteria and recommendations for patient evaluation. Journal of Clinical Endocrinology and Metabolism 2001 86 4041-4046. (doi:10.1210/jc.86.9.4041)

13 Solberg R, Sandberg M, Spurkland A \& Jahnsen T. Isolation and characterization of a human pseudogene for the regulatory subunit RI $\alpha$ of cAMP-dependent protein kinases and its sublocalization on chromosome 1. Genomics 199315 591-597. (doi:10.1006/geno. 1993.1112)

14 Caceres A, Armengol L, Villatoro S \& Gonzalez JR. MLPAstats: an R GUI package for the integrated analysis of copy number alterations using MLPA data. BMC Bioinformatics 201112 147. (doi:10.1186/1471-210512-147)

15 Gonzalez JR, Carrasco JL, Armengol L, Villatoro S, Jover L, Yasui Y \& Estivill X. Probe-specific mixed-model approach to detect copy number differences using multiplex ligation-dependent probe amplification (MLPA). BMC Bioinformatics 20089 261. (doi:10.1186/1471-2105-9-261)

16 Linglart A, Menguy C, Couvineau A, Auzan C, Gunes Y, Cancel M, Motte E, Pinto G, Chanson P, Bougneres P et al. Recurrent PRKAR1A mutation in acrodysostosis with hormone resistance. New England Journal of Medicine 2011364 2218-2226. (doi:10.1056/
17 Diskar M, Zenn HM, Kaupisch A, Prinz A \& Herberg FW. Molecular basis for isoform-specific autoregulation of protein kinase A. Cellular Signalling 200719 2024-2034. (doi:10.1016/j.cellsig.2007. 05.012)

18 Groussin L, Kirschner LS, Vincent-Dejean C, Perlemoine K, Jullian E, Delemer B, Zacharieva S, Pignatelli D, Carney JA, Luton JP et al. Molecular analysis of the cyclic AMP-dependent protein kinase A (PKA) regulatory subunit 1A (PRKAR1A) gene in patients with Carney complex and primary pigmented nodular adrenocortical disease (PPNAD) reveals novel mutations and clues for pathophysiology: augmented PKA signaling is associated with adrenal tumorigenesis in PPNAD. American Journal of Human Genetics 200271 1433-1442. (doi:10.1086/344579)

19 Amieux PS, Cummings DE, Motamed K, Brandon EP, Wailes LA, Le K, Idzerda RL \& McKnight GS. Compensatory regulation of RI $\alpha$ protein levels in protein kinase A mutant mice. Journal of Biological Chemistry 1997272 3993-3998. (doi:10.1074/jbc.272.7.3993)

20 Greene EL, Horvath AD, Nesterova M, Giatzakis C, Bossis I \& Stratakis CA. In vitro functional studies of naturally occurring pathogenic PRKAR1A mutations that are not subject to nonsense mRNA decay. Human Mutation 200829 633-639. (doi:10.1002/humu.20688)

21 Meoli E, Bossis I, Cazabat L, Mavrakis M, Horvath A, Stergiopoulos S, Shiferaw ML, Fumey G, Perlemoine K, Muchow M et al. Protein kinase A effects of an expressed PRKAR1A mutation associated with aggressive tumors. Cancer Research 200868 3133-3141. (doi:10.1158/0008-5472. CAN-08-0064)

Received 10 September 2013

Revised version received 15 October 2013

Accepted 21 October 2013 